\title{
Elemental versus configural perception in a people-present/people-absent discrimination task by pigeons
}

\author{
ULRIKE AUST and LUDWIG HUBER \\ University of Vienna, Vienna, Austria
}

\begin{abstract}
In the present experiment, we investigated whether pigeons rely exclusively on elemental information or whether they are also able to exploit configural information in a people-present/people-absent discrimination task. Six pigeons were trained in a go/no-go procedure to discriminate between 800 color photographs characterized by the presence or absence of people. The people-present stimuli were designated as positive, and the people-absent stimuli were designated as negative. After training and a subsequent generalization test, the pigeons were presented with both familiar and novel people-present stimuli containing human figures that were distorted in one of seven different ways. All the pigeons learned the initial discrimination and also showed generalization to novel stimuli. In the subsequent test, performance on all types of distorted stimuli was diminished in comparison with that on the intact original pictures from which they had been derived. At the same time, however, peck rates clearly exceeded the level of responding found for regular people-absent stimuli. This result strongly suggests that responding was controlled by both the constituting target components and their spatial relations and, therefore, points to the dual importance of elemental and configural information.
\end{abstract}

In previous experiments, we showed that in a peoplepresent/people-absent discrimination task pigeons actually attended to properties of the target - that is, the human figure (Aust \& Huber, 2001). Furthermore, we found that the birds made use of a polymorphous class rule involving collections of differently weighted target features (Aust \& Huber, 2002). However, our research did not uncover whether the pigeons perceived the depicted human figures simply as compounds of unrelated, independent features associated with reinforcement or whether they also attended to the spatial arrangement of the constituting target components.

In humans, the available empirical evidence indicates that the overall or global configuration of a stimulus is typically processed before the local elements (the global precedence effect; Navon, 1977, 1981, 1983). The extent to which animals exploit global, or object-like, properties of stimuli in comparison with more local, or elemental, aspects is still a matter of ongoing research.

Experiments with baboons have shown that, unlike humans, they have a greater facility for processing at the local stimulus level than at the global one. The processing of global information turned out to demand great attention

The research was supported by Grant P14175 from the Austrian Science Foundation. Thanks are due to Michaela Loidolt, Wilfried Apfalter, Martin Fieder, Daniela Seebacher, Andrzej Szpetkowski, and Michael Pollirer for their assistance in the pigeon laboratory. Correspondence should be addressed to L. Huber, Institute of Zoology, University of Vienna, Althanstrasse 14, 1090 Vienna, Austria (e-mail: ludwig.huber@ univie.ac.at). from baboons, whereas humans detect the global target without focal attention. Experiments with chimpanzees as well as those with baboons also failed to reveal an advantage to processing at the global stimulus level. But unlike baboons, processing at the local stimulus level was found to demand sustained attention from chimpanzees (Fagot \& Tomonaga, 1999; Fagot, Tomonaga, \& Deruelle, 2001). In summary, the results suggest the existence of differences between nonhuman primate species in the computation of global and local information as well as a phylogenetic trend in the way compound stimuli are processed.

The question of whether pigeons do exclusively rely on elemental information or are also able to use configural cues is one of the fundamental issues in the study of pigeon visual categorization and has occasioned considerable controversy among researchers. Particulate feature theory (PFT), a version of the feature model, purports that pigeons recognize objects by means of "particulate perception" (Cerella, 1986; see also Herrnstein, 1984). According to PFT, pigeons represent objects as random, unorganized collections of local features and are incapable of using more global, organizational properties (e.g., 3-Dstructure or overall spatial arrangement; see KirkpatrickSteger, Wasserman, \& Biederman, 1998). That view originated with a famous experiment by Cerella (1980), in which pigeons were trained to discriminate pictures of Charlie Brown from other characters of the comic strip Peanuts. It turned out that the birds generalized roughly equally to partial or obstructed views of Charlie Brown as well as to scrambled or inverted views. The results indicated that the critical features were local and that their absolute or rela- 
tive placement within the image was irrelevant-that is, relations among features were not encoded. Cerella (1986) claimed that the pigeons saw Charlie Brown as just a pattern that could be subdivided or inverted without losing any of its essence; that is, they saw only "bits and pieces with no comprehension of the whole" ( $\mathrm{p}$. 432). The results of several other studies are consistent with PFT, indicating that pigeons do not attend to the spatial arrangement of features (e.g., Cerella, 1977; Delius \& Hollard, 1995; Towe, 1954). Recently, however, solid evidence has emerged that configural information can be critical in discriminative control not only in humans but also in pigeons and other birds (Brown \& Dooling, 1993; Kirkpatrick-Steger, Wasserman, \& Biederman, 1996, 1998; Steele, 1990; Van Hamme, Wasserman, \& Biederman, 1992; Wasserman, KirkpatrickSteger, Van Hamme, \& Biederman, 1993; Watanabe, 2001; Watanabe \& Ito, 1991; see also Cook, 2001, and Kirkpatrick, 2001).

In the face of these contrasting results, it appears that pigeons, much like humans, can discriminate complex stimuli on the basis of elemental as well as configural information and can flexibly switch processing between these different spatial scales. However, how and why these different types of stimulus control develop is still poorly understood. Among other factors, processing appears to depend on properties of the avian visual system, attentional factors, and stimulus factors such as feature salience, motion, viewing distance, and configural organization (Cook, 2001). Regarding the latter factor, Riley (1984), for example, noted that decomposition (i.e., local processing) may be more likely to occur in pigeons if the various parts of a compound stimulus can be readily analyzed into different figural components, so that each of the relevant attributes is a component of a different figure. On the other hand, decomposition might be less likely to occur if the relevant attributes are components of the same figure.

Furthermore, the way in which stimuli are processed in a particular visual task has repeatedly been shown to depend on stimulus material (Herrnstein, 1985; Huber, Aust, Michelbach, Ölzant, \& Nowotny, 1999). A key attribute of the experiment by Cerella (1980) was the use of highly artificial stimuli (i.e., line drawings). However, along with the advantages of such stimuli-simple manipulation and feature control-come some cautions. First, through schematization, information that may be crucial for comprehension is lost (Cerella, 1982). Second, pigeons' perception of line drawings appears to differ from ours (Cerella, 1982). Finally, naturalistic images engage perceptual processes not elicited by line projections (Watanabe, 2001). Therefore, it is conceivable that the pigeons' limitations in recognizing the structure of the Peanuts images were due to the use of line drawings and may be different with color stimuli. This does not necessarily imply that pigeons are actually able to recognize real objects in photographs-a question which still awaits a definite answer (see, e.g., Dawkins, Guilford, Braithwaite, \& Krebs, 1996; Delius, Emmerton, Hörster, Jäger, \& Ostheim, 2000; Fagot, MartinMalivel, \& Dépy, 2000; Lea \& Dittrich, 2000; Ryan \& Lea, 1994; Watanabe, 1993, 2000; for a review, see Bovet \&
Vauclair, 2000). But, possibly, the composition and structure of such stimuli may come closer than do line drawings to what pigeons encounter in their environments and what their visual system is prepared to process. In fact, Watanabe (2001) demonstrated that the suppressing effect of scrambling was stronger for more "real" stimuli (photographs) than for "unreal" ones (cartoons) when pigeons had to discriminate slides of a particular person from those of other persons, and slides of pigeons from those of other birds.

The aim of the present study was to test whether pigeons could make use of configural target properties in a people-present/people-absent discrimination task when naturalistic color photographs were used as stimuli. The experiment reported here differs from that of Cerella (1980) as well as from those by Kirkpatrick-Steger et al. $(1996,1998)$ and Wasserman et al. (1993), since those authors all employed line drawings and (with the exception of Cerella, 1980) pictures of objects rather than of persons. The study by Watanabe (2001), who used color photographs of persons and of birds, was concerned with a somewhat different topic —namely, a direct comparison between "natural" and cartoon stimuli. Therefore, his experimental design, the stimuli employed, and the conclusions drawn from his results deviate clearly from ours.

In the present experiment, the birds were first trained to discriminate between pictures containing intact human figures and pictures that were devoid of humans. Then, they were subjected to a generalization test to make sure that learning was based on more than just memorization of item-specific stimulus properties. In a subsequent elemental-configural(EC) test, the birds were confronted with both familiar and novel people-present stimuli, containing human figures that were distorted in one of seven different ways. Two contrasting predictions were available for this experiment: (1) If pigeons rely on target components only, irrespective of their relative and absolute position, performance should not be affected by the presentation of "pathological" instances; and (2) if, on the other hand, the internal target representations also entail configural information such as the spatial arrangement of the constituting features, performance decrements should occur when those interrelations are altered. Sensitivity to spatial relations between features should allow pigeons to discriminate between pictures that are composed of the same components but have different organizations. Consequently, distorted versions should be discriminable from the intact originals.

At first sight, the experimental design seems to bear some similarities to that of Experiment 2 of Aust and Huber (2001), with both involving the presentation of scrambled stimuli. On closer inspection, however, these similarities turn out to be only superficial. Aust and Huber (2001, Experiment 2) examined the role of local versus global information regarding the entire stimulus (without distinguishing between information contained in the target and that contained in the background). By contrast, in the present experiment we examine the importance of elemental versus configural information contained in the 
target only. These different focuses are reflected by clearly distinct modes of scrambling. Whereas in the former experiment targets as well as backgrounds were scrambled, in the present one distortions were applied to targets only. Therefore, only the present experiment allows for conclusions about the role of target integrity in classification, as only here did irrelevant background information remain unaltered. Furthermore, the stimuli in Aust and Huber (2001) were destroyed gradually and according to only one specific algorithm, resulting in progressively smaller and more numerous squares. Thus, the test stimuli differed from each other mainly in the degree of scrambling. In the present experiment, however, each stimulus was scrambled in seven qualitatively different modes. Through this procedure, we expected to gain some insight into the nature of the spatial relations that may be relevant to target identification.

\section{METHOD}

\section{Subjects}

We employed 6 pigeons of a local Austrian race (Columba livia Strasser) as experimental subjects. All the birds were familiar with the experimental chamber and the procedure at the onset of the experiment, since they had already served as subjects in previous experiments (e.g., Troje, Huber, Loidolt, Aust, \& Fieder, 1999). They were, however, naive with respect to the task of classifying pictures according to the presence or absence of people. Food was provided only during and immediately after the experimental sessions. Water and grit were freely available in the aviary. The birds were maintained at about $90 \%$ of their free-feeding weights.

\section{Stimuli}

The stimuli were color photographs taken from the database of the Photodisc Starter Kit and the Photodisc collection (www.photodisc. com). Half of the stimuli used during training and in the generalization tests showed one or more people, whereas the other half were devoid of humans or human body parts. The pictures included a wide variety of settings, and the depicted persons varied in number, identity, sex, race, age, size, apparel, position within the picture, posture, and the amount of area of the picture that they covered. Figure 1 displays some representative samples of the photographs used during training (A) and in the generalization test (B).

The test stimuli used in the EC test were derived from 80 regular, intact people-present stimuli, half of which were familiar training stimuli (Original- $f$ ), whereas the other half were novel (Original $-n)$. The intact originals consisted of a human figure that had been pasted onto a people-absent background. Figure 1C presents an example of how the intact originals were created. In each of the 80 original stimuli the human figure was manipulated in various ways, which all involved unusual orientation and/or "pathological" rearrangements of parts. In particular, seven different test stimuli were derived from each intact original. Figure $2 \mathrm{~A}$ shows a schematically drawn intact (original) human figure as well as its various types of distortion. Figure 2B displays a realistic example of all types of test stimuli, as well as the corresponding intact original. In detail, the test stimuli were created as follows.

Attached. The human figure was cut into head, trunk, first arm, second arm, first leg, and second leg. Then, the parts of the body were reconnected in a disorganized manner. No part was spatially separated from the others, and the orientation of the individual parts remained unaltered.

Detached. The human figure was cut into the same parts as in the attached condition. The parts were then randomly distributed over the entire picture in such a way that no part of the body adjoined to any other part. The orientation of the individual parts remained unaltered.
A)

$\mathbf{P}$
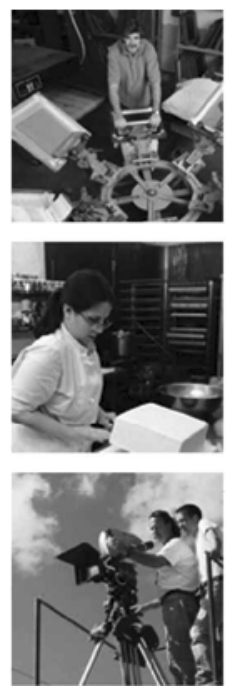

B)

$\mathbf{P}$
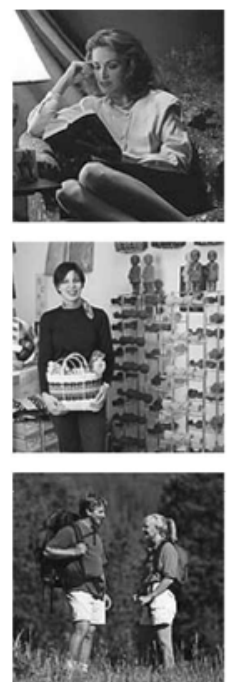

C)

\section{NP}
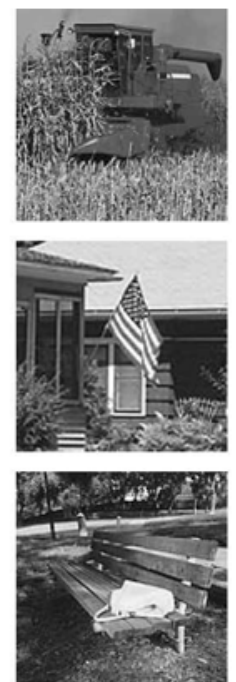

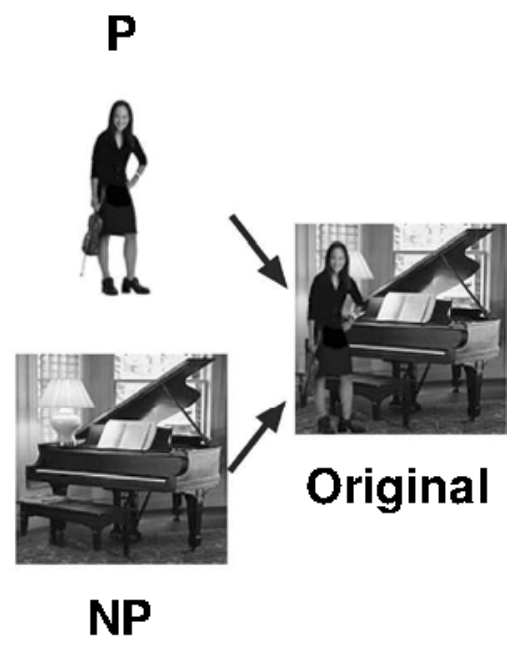

Figure 1. (A) Examples of training stimuli of the people-present (P) and people-absent (NP) classes. (B) Examples of transfer stimuli of both classes shown in the generalization test. (C) Intact original stimuli were created by pasting a human figure (P) onto a people-absent background (NP). The stimuli were presented as full-color photographs. 
Horizontal. The human figure was trisected horizontally into head, torso, and legs. The parts were then reassembled in such a way that they were stuck together vertically, but no adjacent parts were in their proper biological order. Each part retained its right-side-up orientation.

Vertical. The human figure was cut vertically into halves. Whereas one half remained unaltered, the other was rotated by $180^{\circ}$. Then the two halves were reassembled. Thus, one half retained its right-sideup orientation, whereas the other appeared upside-down.

Inverted. The entire human figure was rotated by $180^{\circ}$ so that it was presented upside-down. Thus, the constituting parts were rearranged with respect to orientation and absolute position in the picture, but their relative positions - that is, the spatial relations among them-remained unaltered.

Square. Six squares of $10 \times 10$ pixels were cut out of the human figure and rearranged in a random fashion within its outlines. In order to fill up the available space, the individual squares had to be used more than once. Very rarely, the human figure was too small to allow six squares to be cut out of it. In those cases, as many squares as possible (e.g., four or five) were created.

Irregular. The entire human figure was copied several times onto itself, in a randomly shifted rather than congruent manner, so that the figure pasted last occluded parts of the copies below it. This generated a collage effect, with the outlines of the human figure being filled with randomly arranged pieces of the human figure. Those pieces were of varying shapes and sizes and, again, appeared more than once each.

\section{Apparatus}

The birds entered an experimental indoor chamber (Skinner box) from an outdoor aviary compartment through a passway system

\section{A) Original}
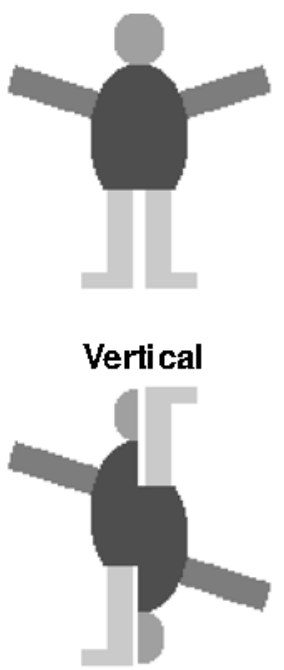

B)

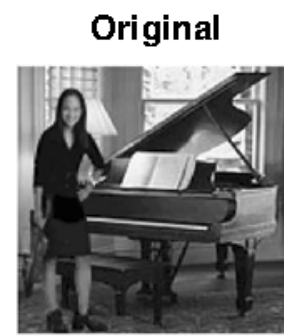

Vertical

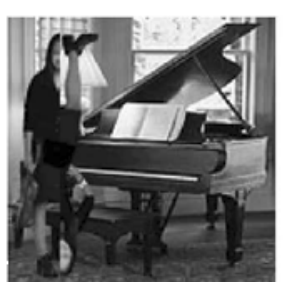

Attached
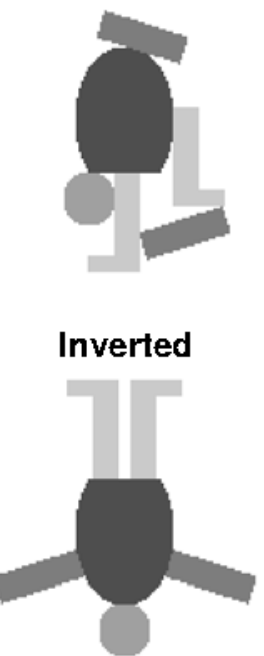

Attached

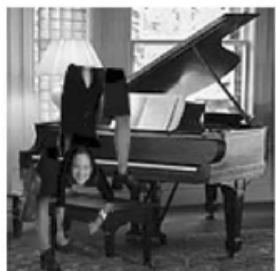

Inverted

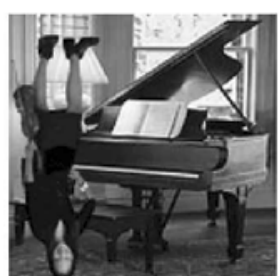

Detached

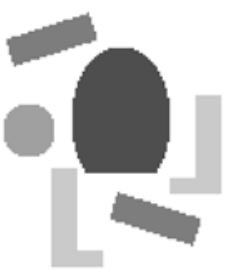

Square

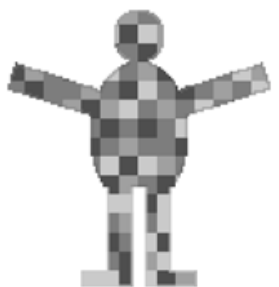

Detached

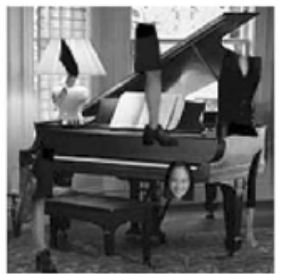

Square

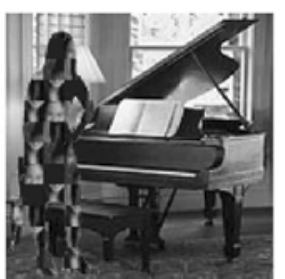

\section{Horizontal}

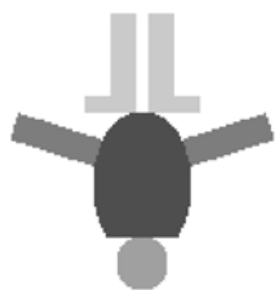

Irregular

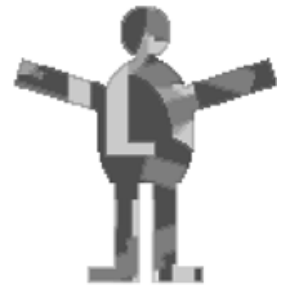

Horizontal

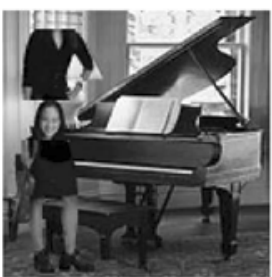

Irregular

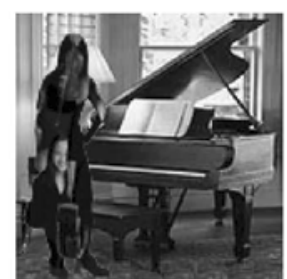

Figure 2. (A) Schematic and (B) realistic examples of the seven different ways in which the original intact human figure contained in the test stimuli was distorted. The stimuli were presented as full-color photographs. 
(Huber, 1994). The interior size of the wooden chamber was $50 \times$ $30 \times 40 \mathrm{~cm}$. In the center of the front panel, there was a clear Perspex pecking key ( $5 \mathrm{~cm}$ diam.; ENV-125M, MED Association, USA). Directly below the key was a $6 \times 6 \mathrm{~cm}$ aperture for a 28 -V DC solenoid activated hopper of the grain feeder (ENV-205M). A hopper light illuminated the receptacle area whenever grain was accessible. Except for a dark intertrial interval (ITI) that preceded stimulus presentation, the chamber was weakly illuminated throughout the experiment by a $2-\mathrm{W}$ house light (ENV-215) located in the rear part of the chamber. Stimulus presentation was accomplished by a Pentium PC running a Delphi program that selected the patterns according to a prespecified sequence. The stimuli were presented on a 15-in. (38$\mathrm{cm}$ diagonal) CRT monitor (Panasonic PanaSync 4G) at a distance of $5 \mathrm{~cm}$ behind the pecking key. They were presented at a size of 128 $\times 128$ pixels at a resolution of $1,024 \times 768$ pixels, thus producing a $45 \times 45 \mathrm{~mm}$ picture on the monitor. The MED for Windows behavioral control package, which was installed on a separate PC, was used to control events in the operant chamber, to signal onset and offset of presentation to the presentation PCs, and to record responses.

\section{Procedure}

All 6 pigeons were familiar with the experimental chamber and the procedure at the onset of the experiment. Thus, they were immediately subjected to the training procedure. The stimuli containing at least one person (Class $\mathrm{P}$ ) were designated positive, and those that did not show any people (Class NP) were designated negative.

We used a standard go/no-go procedure such as that described by Vaughan and Greene (1984; see also Aust \& Huber, 2001; Herrnstein \& Loveland, 1964; Herrnstein, Loveland, \& Cable, 1976), which required the pigeons to peck in the presence of positive stimuli and to refrain from pecking in the presence of negative stimuli. Each bird received one session per day, 5 days a week, with each session consisting of the presentation of 40 stimuli. The entire sequences were quasi-random insofar as they never contained more than 3 positive or 3 negative stimuli in immediate succession, and the first stimulus of each session was always a positive one. The training procedure required the pigeons to discriminate between 20 positive and 20 negative stimuli in each session. Pecks were counted only during the first $10 \mathrm{sec}$ of a trial. During the subsequent variable interval (VI; mean, $10 \mathrm{sec}$; range, $1-20 \mathrm{sec}$ ), it was unimportant whether or not the pigeons pecked, as pecks were not recorded. After the VI was terminated, pecks were recorded again — not for later data analysis, but in order to determine whether the response requirement for positive or negative trials was met. On positive trials, two pecks emitted within $2 \mathrm{sec}$ (after the VI) resulted in $5 \mathrm{sec}$ of food access. On negative trials, each response emitted to a negative stimulus prolonged stimulus presentation, which was terminated only after no further responses had occurred for $8 \mathrm{sec}$. No food was delivered on negative trials. The image remained visible throughout the entire trial. Each trial was followed by an ITI, a 4-sec dark phase that signaled the forthcoming stimulus presentation. Test stimuli were presented with neutral contingencies, which means that the respective test trial resulted neither in food access nor in a delay interval, but was terminated after the first $10 \mathrm{sec}$ of presentation during which pecks were counted. Table 1 provides an overview of the experimental design.

Training. The pigeons were trained to discriminate between stimuli of Class $\mathrm{P}(\operatorname{trP})$ and of Class NP $(\operatorname{trNP})$ in equal numbers. The range of training stimuli was extended in a stepwise fashion. Training Phase 1 involved a total of 320 stimuli. Each day, the pigeons were presented with 40 novel stimuli and had thus seen a complete cycle of all 320 stimuli after eight sessions. The pigeons completed three such cycles, which means that they experienced each stimulus three times. In Phase 2, a total of 160 novel stimuli were presented. Consequently, a complete cycle required four sessions. Again, we ran three complete cycles, which means that each stimulus was shown three times. Training Phase 3 was carried out in exactly the same manner as the previous ones and involved the presentation of another 320 novel stimuli, thus resulting in a total of 800 training stimuli. In a review phase (Phase 4), each of those 800 stimuli was presented one more time. Phase 4 required 20 sessions. In summary, each of the 800 stimuli had been presented four times by the end of training.

Generalization test. The pigeons were then subjected to a generalization test, which consisted of 10 consecutive sessions. Eighty novel nonreinforced stimuli, 40 of Class $\mathrm{P}(t e P)$ and 40 of Class NP $(t e N P)$, were interspersed at a rate of 8 per session into sequences of ordinary training stimuli (trP and trNP). Thus, each test session involved the presentation of 8 test stimuli and 32 training stimuli. The latter were arbitrarily selected from the 800 stimuli introduced during training. The criterion of mastery was defined by the experimenter as $\rho$ (rho) $\geq .700$ for the classification of the 40 positive (teP) versus the 40 negative (teNP) test stimuli. $\rho$ values represent a nonparametric statistic introduced by Herrnstein et al. (1976; see also Aust \& Huber, 2001, 2002). On the basis of a Mann-Whitney $U$ comparison, it is used to estimate the probability that the average positive exemplar is ranked above the average negative exemplar, using ranked rates of pecking as the measure of performance. The chosen criterion was set somewhat above the limit of significance (.626). This was done because in the subsequent EC test, the occurrence of significant performance decrements on distorted in comparison with intact stimuli would presuppose reasonably good baseline performance on the latter. After successfully passing the generalization test, the pigeons were transferred to the EC test.

Elemental-configural (EC) test. Table 2 presents details on the types, numbers, and contingencies of the stimuli shown in the EC test. The test consisted of 60 sessions. As the distorted test stimuli were derived from familiar intact stimuli (Original-f) as well as novel ones (Original-n), the test included two subconditions: Test

Table 1

Overview of the Experimental Design

\begin{tabular}{|c|c|c|c|c|c|c|c|}
\hline & Train & Train/Session & Test & Test/Session & Sessions & Cycles & Sessions/Cycle \\
\hline \multicolumn{8}{|l|}{ Training } \\
\hline Phase 1 & 320 & 40 & & & 24 & 3 & 8 \\
\hline Phase 2 & 160 & 40 & & & 12 & 3 & 4 \\
\hline Phase 3 & 320 & 40 & & & 24 & 3 & 8 \\
\hline Phase 4 & 800 & 40 & & & 20 & 1 & 20 \\
\hline \multicolumn{8}{|c|}{ Generalization Test } \\
\hline & 800 & 32 & 80 & 8 & 10 & 1 & 10 \\
\hline \multicolumn{8}{|c|}{ Elemental-Configural Test } \\
\hline & 920 & $30 *$ & 600 & 10 & 60 & 1 & 60 \\
\hline
\end{tabular}

Note-Train $=$ number of training stimuli; train/session $=$ number of training stimuli per session; test $=$ number of test stimuli; test/session $=$ number of test stimuli per session; sessions $=$ number of sessions required; cycles $=$ number of cycles; sessions/cycle $=$ number of sessions per cycle. $\quad *, 28$ familiar training stimuli (trP-f, trNP-f) plus 2 novel regular instances (trP-n, trNP-n). 
Table 2

Types, Numbers, and Contingencies of the Stimuli Shown in the Elemental-Configural Test

\begin{tabular}{llll}
\hline Stimulus Type & No. & Con & Description \\
\hline trP-f & 400 & tr & Familiar training stimuli of Class P \\
trNP-f & 400 & tr & Familiar training stimuli of Class NP \\
trP-n & 60 & tr & Novel stimuli of Class P \\
trNP-n & 60 & tr & Novel stimuli of Class NP \\
Original-f & 40 & tr & Familiar (intact) stimuli of trP-f, from which half of the test stimuli were derived \\
Original-n & 40 & te & Novel (intact) stimuli, from which the other half of the test stimuli were derived \\
Attached-f & 40 & te & Distorted "attached" version derived from Original-f stimuli \\
Attached-n & 40 & te & Distorted "attached" version derived from Original-n stimuli \\
Detached-f & 40 & te & Distorted "detached" version derived from Original-f stimuli \\
Detached-n & 40 & te & Distorted "detached" version derived from Original-n stimuli \\
Horizontal-f & 40 & te & Distorted "horizontal" version derived from Original-f stimuli \\
Horizontal-n & 40 & te & Distorted "horizontal" version derived from Original-n stimuli \\
Vertical-f & 40 & te & Distorted "vertical" version derived from Original-f stimuli \\
Vertical-n & 40 & te & Distorted "vertical" version derived from Original-n stimuli \\
Inverted-f & 40 & te & Distorted "inverted" version derived from Original-f stimuli \\
Inverted-n & 40 & te & Distorted "inverted" version derived from Original-n stimuli \\
Square-f & 40 & te & Distorted "square" version derived from Original-f stimuli \\
Square-n & 40 & te & Distorted "square" version derived from Original-n stimuli \\
Irregular-f & 40 & te & Distorted "irregular" version derived from Original-f stimuli \\
Irregular-n & 40 & te & Distorted "irregular" version derived from Original-n stimuli \\
\hline
\end{tabular}

Note-No. = total number of stimuli of the respective type; Con $=$ contingency with which the respective stimuli were shown (training: tr; test: te).

Familiar and Test Novel. The 40 familiar intact originals of Test Familiar were arbitrarily selected from the pool of 400 training stimuli of Class P (trP-f). Those stimuli were reinforced in the same manner as the other ordinary instances of Class $\mathrm{P}$ (i.e., they had training contingency). They could appear more than once in the course of the test, because choice of training stimuli-accomplished by the DELPHI program-was random. Each test session involved the presentation of 10 test stimuli selected from the set of 40 Original-n stimuli as well as from the 560 distorted stimuli ( 2 subconditions $\times 40$ stimuli $\times 7$ modifications). They were interspersed with test contingencies into sequences of ordinary familiar training stimuli (trP- $f$ and trNP-f). Furthermore, each session involved the presentation of two novel stimuli that were regular instances of the two classes (trP$\mathrm{n}$ and trNP-n). They were interspersed with training contingencies in order to prevent the pigeons from learning that it was irrelevant whether or not they responded to stimuli recognized as unfamiliar. Thus, each test session involved the presentation of 28 familiar training stimuli, 2 novel stimuli (one of each class), and 10 test stimuli.

\section{Statistics}

Performance on positive versus negative stimuli during training and in the generalization test was assessed in terms of $\rho$ values. That measure was chosen to make the results comparable with those by Herrnstein and Loveland (1964) and those of Aust and Huber (2001, Experiment 2; 2002, training), which were based on ranked data as well.

Assessment of the results of the EC test was based on the mean standardized response rate. This is a more demanding measure than the $\rho$ value and was obtained by dividing the absolute number of pecks emitted on each trial of a session (both training and test trials) by the average peck rate of that session, as measured on trials with training contingencies only. As the data obtained for the EC test failed to show normal distribution, nonparametric statistics were applied. Mann-Whitney $U$ tests (including Bonferroni corrections) were carried out to compare performances on the individual stimulus types and to assess differences in performance between corresponding stimulus types in the two tests (Familiar and Novel) as well as differences among individual birds. To rule out the possibility that responding might have been influenced by the order in which the individual dis- torted versions of a stimulus were presented, Spearman rank correlations between order of presentation and peck rates were calculated.

\section{RESULTS}

Acquisition performance during training appears as $\rho$ values in Figure 3. The pigeons rapidly learned to sort at a significant level, with most of the birds showing some grasp of the underlying class rule within a few sessions. Even the introduction of novel stimuli (Cycle 1 in each phase) caused only little detriment to discrimination, and performance recovered quickly.

Figure 4 presents a summary of the results of the generalization test as mean standardized response rates $( \pm S D)$, shown in comparison with the first presentation of the training stimuli. The means were taken across the 6 birds. The figure indicates that the birds performed excellently on the test stimuli. This finding was confirmed by the corresponding $\rho$ values, which were calculated separately for the training and test components. Calculations of the training component were based on the peck rates on the first presentation of each of 80 randomly selected training stimuli shown during the test. Regarding the training component, the achieved $\rho$ values (averaged across all birds; value needed to assign significance: .626) were .782 , and all 6 birds achieved $\rho \geq .700$ (i.e., the criterion of mastery defined by the experimenter). On the test component, the respective $\rho$ value (again averaged across all birds) was .809. In fact, all 6 birds passed the generalization test without difficulty.

Figure 5 illustrates the results of the EC test. It depicts the data obtained for the individual types of test stimuli in both Test Familiar and Test Novel as well as those obtained for the respective intact original stimuli as mean stan- 


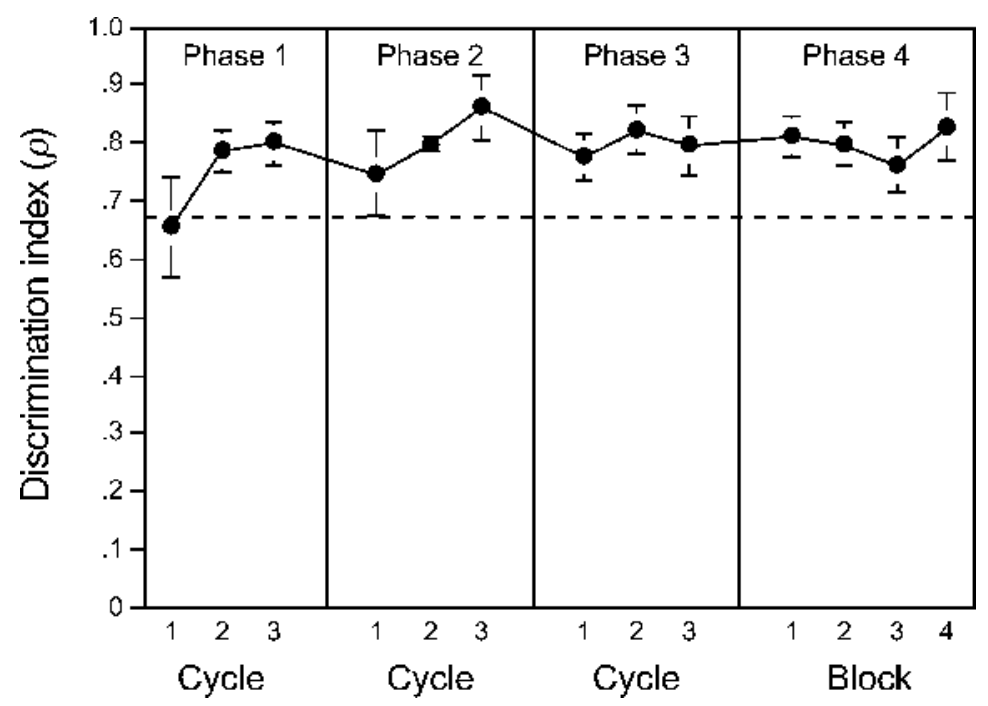

Figure 3. Performance during the individual phases of training, shown as $\rho$ values $( \pm S D)$. The means were taken across the 6 birds. The dashed line indicates the limit of significance (.680). Phases 1,2 , and 3 consisted of three cycles each, which comprised eight sessions in Phases 1 and 3 and 4 sessions in Phase 2. Phase 4 consisted of 20 sessions, which are depicted in blocks of five.

dardized response rates $( \pm S D)$. In Test Familiar, each of the intact originals (Original-f) appeared more than once, but only the first presentation of each picture in the test was taken into account. Figure 5 also shows performance on the first presentation of 60 arbitrarily chosen familiar training stimuli of each class (i.e., stimuli taken from the pools of trP-f and trNP-f ), as well as on the 60 novel regular instances of each class (trP-n and trNP-n), which had been interspersed with training contingencies at a rate of two per session.

Baseline performance was assessed by comparing peck rates on the individual training components (trP-f, trNP-f, trP-n, and trNP-n) and on the intact original stimuli (Original-f, Original-n) using a Mann-Whitney $U$ statistic. As the Bonferroni procedure was applied, the $\rho$ value needed to assign significance was .0083. Because there was not much variance among individual birds-only 6 of 180 comparisons $(2$ tests $\times 6$ stimulus types $\times 15$ bird combinations) indicated significant differences - calculations were based on means taken across experimental subjects. Discrimination performance on the first presentation of positive and negative familiar training stimuli (trP-f and trNP-f) was extraordinarily good $(p \leq .0001)$. As regards the novel regular stimuli of Class $\mathrm{P}(\operatorname{tr} \mathrm{P}-\mathrm{n})$, the birds did even better than they had on the familiar training stimuli (trP-f; $p=.0069$ ). We did not find any significant differences between performance on either of the intact originals (Original-f or Original-n) from which the test stimuli had been derived and that on the familiar training stimuli of Class P (trP-f). Whereas performance on the novel regular stimuli of Class P (trP-n) exceeded that on the intact familiar originals (Original-f; $p \leq .0001$ ), it did not deviate from performance on the intact novel originals (Original-n).
Furthermore, statistical analysis revealed no significant difference between familiar and novel intact originals (Original-f and Original-n).

Regarding performance on the distorted test stimuli, the individual birds, in general, responded quite similarly to each of the seven types. Only 16 comparisons of 210 ( 2 tests $\times 7$ stimulus types $\times 15$ bird combinations) revealed significant interindividual differences. So, again, all further calculations were based on peck rates averaged across experimental subjects.

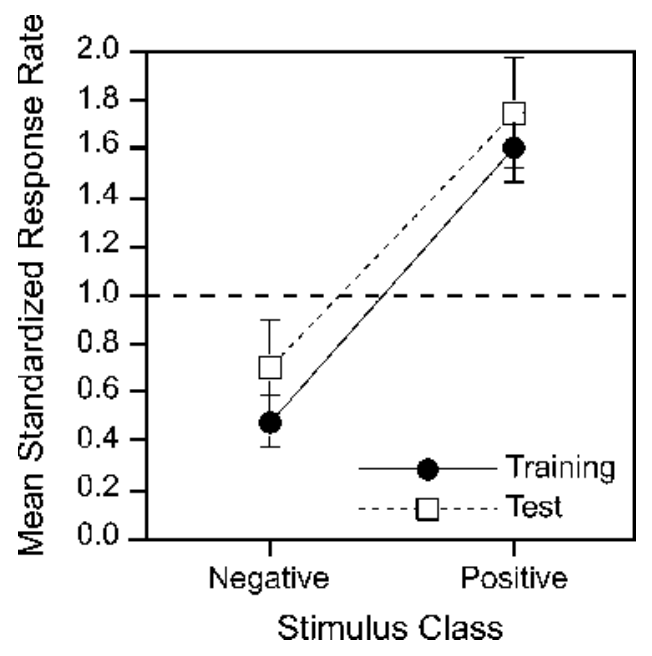

Figure 4. Generalization performance, shown as mean standardized response rate $( \pm S D)$, in comparison with the first presentation of 80 arbitrarily selected training stimuli during the test. The means were taken across all birds. 


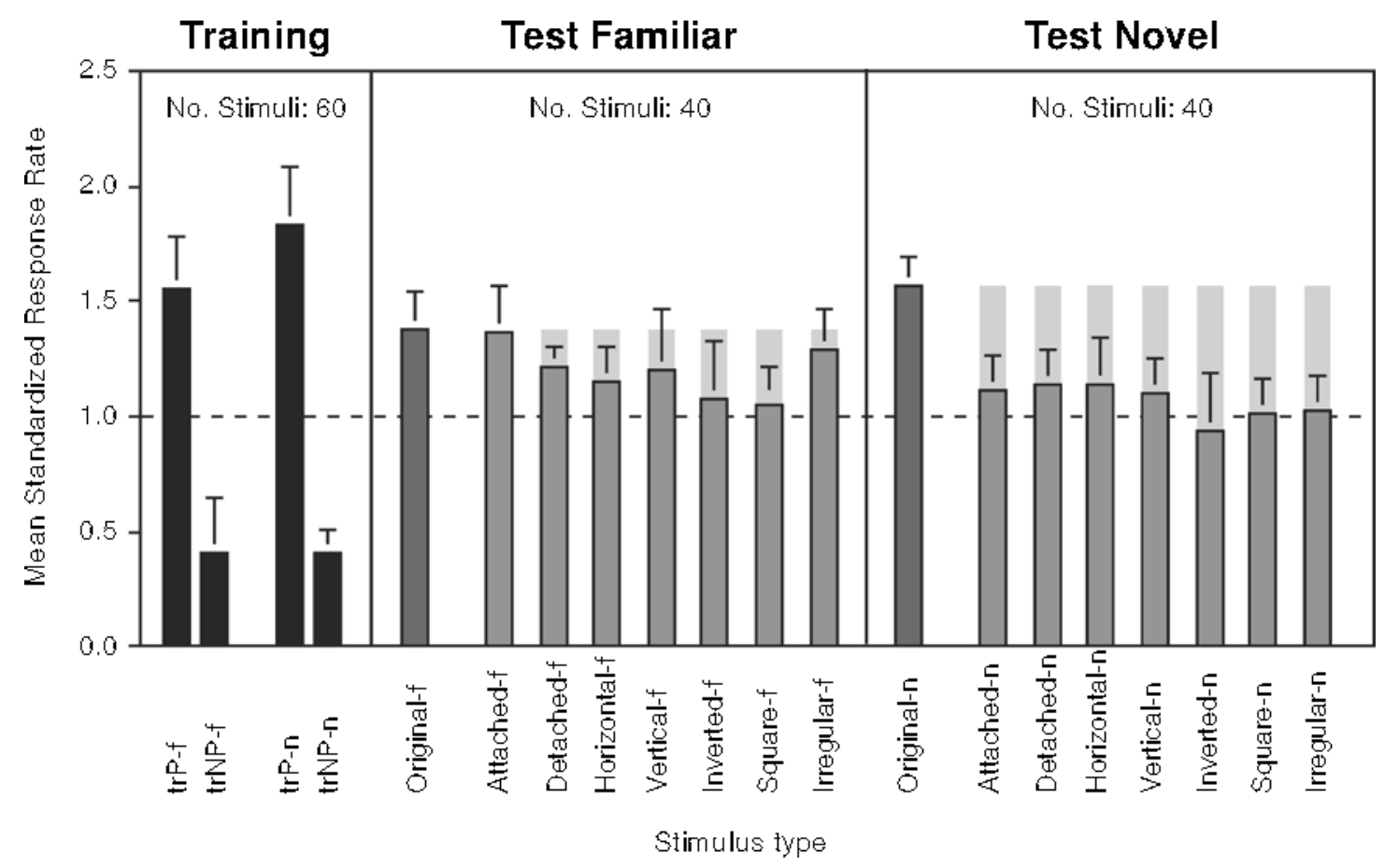

Figure 5. Results of the elemental-configural test, shown separately for Test Familiar and Test Novel as mean standardized response rates. The black columns in the left-hand section show performance on the first presentation of 60 arbitrarily chosen familiar training stimuli of both classes (trP-f, trNP-f) and on the novel regular stimuli (trP-n, trNP-n). The gray columns of the middle and right-hand sections show performance on the seven types of test stimuli in Tests Familiar and Novel, respectively, together with their respective intact originals, represented by dark gray columns. The light gray shades prolong the columns of the test stimuli up to the level of their intact originals to make the respective declines in performance more obvious.

Performances on the individual types of stimuli (intact originals and distorted derivatives) were compared by means of a Mann-Whitney $U$ test (needed $p$ value after Bonferroni correction: .0063). The results are summarized in Table 3. Figure 5 indicates that all types of distortion (except Attached-f) led to performance decrements in comparison with the respective intact originals in both tests. However, a statistical analysis revealed that in Test Familiar, none of those differences was significant. By contrast, in Test Novel, performance on the intact original stimuli (Original-n) deviated significantly from performance on the distorted test versions in all but one case (Detached-n). For neither test did we find significant differences among any of the seven individual types of test stimuli.

In order to rule out the possibility that reduced responding to distorted stimuli was merely a consequence of inadvertent image artifacts introduced by scrambling (e.g., high-frequency noise in the form of added contrast boundaries), it was necessary to investigate whether peck rates were influenced by the serial order in which each of the test stimuli appeared in its eight guises. Spearman rank correlations calculated between peck rates and order of presentation were nonsignificant for all the birds in both tests, indicating that the pigeons actually did not pick up on such foreign image elements.
Furthermore, it is important that performance on distorted stimuli-although diminished in comparison with the intact originals-never dropped to the level of responding found for regular instances of Class NP. Differences between peck rates on trNP-f and trNP-n on the one hand, and on the test stimuli on the other, were significant for all test stimulus types in both tests (Familiar and Novel), the $p$ value needed to assign significance after Bonferroni correction being .0056. The results are presented in detail in Table 4.

\section{DISCUSSION}

The outcome of the present experiment allows for the following conclusions. First, it is apparent from the results that discriminative responding was controlled by more than particulate aspects and that a feature-only account is thus insufficient to describe the pigeons' target representation. Overall, the pigeons responded less-in Test Novel even significantly less - to the distorted test versions than to the intact originals from which these had been derived (see Table 3). As inadvertent image artifacts introduced by scrambling could be ruled out as a possible alternative reason for that decline, the results actually reflect the importance of relational information. Nevertheless, peck 
Table 3

Comparison of Performance on Intact Originals and Distorted Derivatives

\begin{tabular}{|c|c|c|c|c|c|c|c|}
\hline & Orig. & Att. & Det. & Hor. & Ver. & Inv. & Sq. \\
\hline \multicolumn{8}{|c|}{ Test Familiar } \\
\hline Att. & .9463 & & & & & & \\
\hline Det. & .2181 & .2602 & & & & & \\
\hline Hor. & .1102 & .1168 & .5834 & & & & \\
\hline Ver. & .2253 & .2562 & .9693 & .5508 & & & \\
\hline Inv. & .0075 & .0141 & .1237 & .4764 & .1333 & & \\
\hline $\mathrm{Sq}$. & .0108 & .0237 & .1410 & .4705 & .1260 & .7950 & \\
\hline Irr. & .4824 & .5254 & .5768 & .3457 & .6304 & .0567 & .0675 \\
\hline \multicolumn{8}{|c|}{ Test Novel } \\
\hline Att. & .0032 & & & & & & \\
\hline Det. & .0114 & .9081 & & & & & \\
\hline Hor. & .0033 & .9310 & .9386 & & & & \\
\hline Ver. & .0024 & .5254 & .5720 & .5508 & & & \\
\hline Inv. & $\leq .0001$ & .0766 & .0960 & .0675 & .1842 & & \\
\hline $\mathrm{Sq}$. & .0002 & .3170 & .2685 & .2522 & .6720 & .5191 & \\
\hline Irr. & .0007 & .4884 & .4471 & .3657 & .8323 & .1939 & .9081 \\
\hline
\end{tabular}

Note-Differences between performance on the intact originals and that on the seven types of test stimuli in Tests Familiar and Novel are given as $p$ values as revealed by Mann-Whitney $U$ tests. The means were taken across the 6 birds. $P$ value needed to assign significance: .00625 . Significant $p$ values are presented in italics. Orig. = original; Att. = attached; Det. = detached; Hor. = horizontal; Ver. = vertical; Inv. = inverted; Sq. = square; Irr. = irregular.

rates were significantly higher on the test stimuli than on regular instances of Class NP (trNP-f and trNP-n; see Table 4). This indicates that responding was, to a great extent, based on elemental features that were still present in the distorted stimuli. In summary, the level of performance on the distorted test stimuli is consistent with the notion that, on the one hand, the birds must have recognized that the parts of the depicted targets were not in their proper spatial concatenation, but on the other hand, they also recognized that the distorted versions contained the same elements as the intact originals. This suggests an important role of both componential features and their spatial organization (see Kirkpatrick-Steger et al., 1996, 1998; Steele, 1990; Wasserman et al., 1993; Watanabe, 2001; Watanabe \& Ito, 1991).

Second, performance decrements were significant only when the originals were novel (Original-n). Since there was no significant difference between performance on familiar and novel originals, this finding suggests that responding to the test stimuli was influenced by item-specific information in Test Familiar. As has been argued elsewhere (Aust \& Huber, 2001; Loidolt, Aust, Meran, \& Huber, 2003), pigeons are able to extract and store both item-specific and category-specific information during training. However, they use only category-specific information to determine category membership of scrambled versions of novel items, whereas item-specific features that survive truncation are the main source of information in the categorization of scrambled versions of familiar stimuli. Accordingly, in Test Familiar of the present experiment, loss of configural integrity could be compensated to some extent by item-specific information, acquired by experience with the training stimuli. However, the fact that also in Test Familiar there was at least some drop in performance in most cases (see Figure 5) indicates that dis- crimination strategies entailing configural knowledge were not completely absent.

In the case of Test Novel, the pigeons had no possibility of compensating for the deficiencies in the spatial arrangement of target elements by falling back on itemspecific information, but, instead, had to rely on categoryspecific information. The stimuli of both Tests Familiar and Novel provided correct elemental cues but invalid configural cues (i.e., the pictures contained all the target components, but these did not appear in their proper anatomical arrangement). The fact that performance was more strongly affected in Test Novel than in Test Familiar suggests a more critical role of configural information in cases in which no item-specific elemental cues are available than when presence of the latter provides a reliable alternative basis for discrimination.

Table 4

Comparison of Performance on Intact Instances of Class NP and Test Stimuli

\begin{tabular}{lrrrrr}
\hline & \multicolumn{2}{c}{ Test Familiar } & & \multicolumn{2}{c}{ Test Novel } \\
\cline { 2 - 3 } \cline { 5 - 6 } & trNP-f & trNP-n & & trNP-f & trNP-n \\
\hline Att. & $\leq .0001$ & .0003 & & $\leq .0001$ & .0010 \\
Det. & $\leq .0001$ & .0005 & & $\leq .0001$ & .0007 \\
Hor. & $\leq .0001$ & .0007 & $\leq .0001$ & .0009 \\
Ver. & $\leq .0001$ & .0008 & & $\leq .0001$ & .0009 \\
Inv. & .0002 & .0014 & & .0006 & .0057 \\
Sq. & .0001 & .0013 & & .0001 & .0018 \\
Irr. & $\leq .0001$ & .0004 & $\leq .0001$ & .0017 \\
\hline
\end{tabular}

Note-Differences between performance on regular instances of Class NP and the seven types of test stimuli in Tests Familiar and Novel are given as $p$ values as revealed by Mann-Whitney $U$ tests. The means were taken across the 6 birds. $P$ value needed to assign significance: .00556. All $p$ values are significant. trNP-f $=$ familiar training stimuli of Class NP; trNP-n = novel stimuli of Class NP; Att. = attached; Det. $=$ detached; Hor. $=$ horizontal; Ver. $=$ vertical; Inv. = inverted; Sq. $=$ square; Irr. = irregular. 
Third, regarding the fact that statistical analysis failed to reveal any significant differences between individual types of test stimuli, general conclusions about the effects of a specific distortion mode have to be approached with some caution. Nevertheless, in Test Novel (in which performance was not contaminated by the effects of itemspecific information), we may at least find some indication that different types of test stimuli were treated in a different manner. For example, the Square-n and Irregular-n stimuli, with their high degree of destruction regarding the spatial relations among target elements, led to a drop in performance down to an intermediate level of responding (about 1.00; see Figure 5). Such ambiguous pecking behavior may reflect the birds' uncertainty about class membership of stimuli containing only a few intact configural cues.

Furthermore, the birds showed a tendency to reject Inverted-n stimuli as regular instances of Class P. This suggests that, in addition to elemental and configural cues, correct orientation of the target figure and/or its constituting elements may have played a role as well. This result is of particular interest with respect to the long-standing question of rotational invariance. Actually, there is evidence of substantial, but not complete, rotational invariance in pigeons. Although they are able to generalize to rotated stimuli, changes in orientation result in accuracy decrements, which depend on the degree of rotation (Cerella, 1977; Herrnstein, 1985; Hollard \& Delius, 1982; Huber \& Aust, 2001; Kirkpatrick, 2001; Towe, 1954; Wasserman et al., 1996). The presentation of a picture upside-down (i.e., rotated by $180^{\circ}$ ), as was the case with the human figures in the inverted condition of the present experiment, means maximum deviation from familiar upright presentation and should thus represent the orientation leading to maximum performance decrements. The present data support that notion, with performance on inverted human figures lying even slightly below the mean level of responding (1.00; see Figure 5).

In some respects, the present results confirm and extend those of Experiment 2 of Aust and Huber (2001), in an investigation of the role of global versus local information. In both cases, we found that scrambling resulted in a reduction of discrimination ability and that the effect of scrambling was stronger with novel than with familiar stimuli. However, only the present experiment allowed conclusions to be drawn about the role of target integrity for classification, since in Aust and Huber (2001) the entire picture was scrambled, whereas in the present study only targets were affected by distortions. This as well as some other differences in experimental design were reflected in the different outcomes of the two experiments. Whereas in Aust and Huber (2001) we found support for basing the classification of scrambled stimuli on small and local features but not for the use of more global ones (although this possibility could not be explicitly ruled out), the results of the present experiment strongly suggest an important role of configural in addition to elemental information.

To return to the studies of Cerella (1980) and Watanabe (2001) and to the question of whether and in what manner the choice of stimulus material may affect the internal target representation, it can be concluded that the use of color photographs instead of line drawings has in fact a facilitating effect on the accessibility of configural information. Whereas with line drawings discrimination seems to be mediated by the specific features of the drawings rather than by the overall relations among their component parts, information contained in color photographs appears to promote partial control by the spatial configuration of the target elements. Actually, our results stand in contrast to those of Cerella (1980), who used line drawings, and are in keeping with those of Watanabe (2001), who also used color photographs.

But what may cause that superiority of "natural" stimuli over "artificial" ones in facilitating the use of configural information? A rather sophisticated explanation would be that pigeons recognize color photographs (but not strongly impoverished pictures such as line drawings) as representations of real objects (i.e., persons). From everyday life experience, they know that the individual parts of human beings always occur in a particular order, and they are confused when this order is violated. However, it is questionable that pigeons are able to see the correspondence between live persons and their 2-D representations. This is a task that makes high demands on a subject's cognitive abilities, and it would tax the pigeons' capacities severely, to say the least. Even evidence with nonhuman primates is ambiguous and inconsistent (see, e.g., Butler \& Woolpy; 1963; Jitsumori, 1991; Winner \& Ettlinger, 1978), and it has been shown that humans also have difficulty recognizing familiar persons in photographs when they are inexperienced with pictures in general (Miller, 1973).

A more modest account would hold that the effect of stimulus material on the accessibility of configural information has to do with the different amounts of information provided by line drawings and color photographs. In the case of line drawings, the "features" are usually nothing more than differently shaped black lines enclosing homogeneously white areas. By contrast, color photographs contain a wealth of information in the form of shapes, textures, and colors. This gives rise to a variety of easily distinguishable areas (or features). Color, for example, has been found to contribute to the ability to structure a picture by setting boundaries (see Aust \& Huber, 2001; Jacobs, 1993; Mollon, 1989; Wurm, Legge, Isenberg, \& Luebker, 1993). As a consequence, the spatial relations among features may become more salient (and thereby more unique and noninterchangeable) than those between the more uniform black and white areas found in line drawings. In short, the accessibility of configural information depends on the detectability of specific spatial relations, which, in turn, may be increased by very distinctive and salient constituting features.

Overall, the results of the present study have shown once more how critically failure or success in pigeon visual categorization experiments may depend on adequate methodology. We have presented evidence of the effect of 
appropriate stimulus material on the formation of target representations and on the strategies chosen by pigeons to cope with a people-present/people-absent discrimination task. In particular, it has been demonstrated that the extraction of configural information is encouraged by the presentation of color photographs instead of line drawings. Whether the use of "natural" instead of "artificial" stimuli may also be the key to success in other visual categorization problems-especially in tasks that have so far been considered difficult or even unsolvable for pigeonsis a question we hope eventually to answer.

\section{REFERENCES}

Aust, U., \& Huber, L. (2001). The role of item- and category-specific information in the discrimination of people versus nonpeople images by pigeons. Animal Learning \& Behavior, 29, 107-119.

Aust, U., \& Huber, L. (2002). Target-defining features in a "peoplepresent/people-absent" discrimination task by pigeons. Animal Learning \& Behavior, 30, 165-176.

Bovet, D., \& VAUClair, J. (2000). Picture recognition in animals and humans. Behavioural Brain Research, 109, 143-165.

Brown, S. D., \& Dooling, R. J. (1993). Perception of conspecific faces by budgerigars (Melopsittacus undulatus): II. Synthetic models. Journal of Comparative Psychology, 107, 48-60.

Butler, R. A., \& Woolpy, J. H. (1963). Visual attention in the rhesus monkey. Journal of Comparative Physiology \& Psychology, 56, 324328.

Cerella, J. (1977). Absence of perspective processing in the pigeon. Pattern Recognition, 9, 65-68.

Cerella, J. (1980). The pigeon's analysis of pictures. Pattern Recognition, 12, 1-6.

Cerella, J. (1982). Mechanisms of concept formation in the pigeon. In D. J. Ingle, M. A. Goodale, \& R. J. W. Mansfield (Eds.), Analysis of visual behavior (pp. 241-249). Cambridge, MA: MIT Press.

Cerella, J. (1986). Pigeons and perceptrons. Pattern Recognition, 19, 431-438.

Cook, R. G. (2001). Hierarchical stimulus processing in pigeons. In R. G. Cook (Ed.), Avian visual cognition [on-line]. Retrieved from www.pigeon.psy.tufts.edu/avc/cook/.

Dawkins, M. S., Guilford, T., Braithwaite, V. A., \& Krebs, J. R. (1996). Discrimination and recognition of photographs of places by homing pigeons. Behavioural Processes, 36, 27-38.

Delius, J. D., Emmerton, J., Hörster, W., Jäger, R., \& Ostheim, J. (2000). Picture-object recognition in pigeons. In J. Fagot (Ed.), Picture perception in animals (pp. 1-35). Hove, U.K.: Psychology Press.

Delius, J. D., \& Hollard, V. D. (1995). Orientation invariant pattern recognition by pigeons (Columba livia) and humans (Homo sapiens). Journal of Comparative Psychology, 109, 276-290.

Fagot, J., Martin-Malivel, J., \& DéPy, D. (2000). What is the evidence for an equivalence between objects and pictures in birds and nonhuman primates? In J. Fagot (Ed.), Picture perception in animals (pp. 295-320). Hove, U.K.: Psychology Press.

FAgot, J., \& Tomonaga, M. (1999). Global and local processing in humans (Homo sapiens) and chimpanzees (Pan troglodytes): Use of a visual search task with compound stimuli. Journal of Comparative Psychology, 113, 3-12.

Fagot, J., Tomonaga, M., \& Deruelle, C. (2001). Processing of the global and local dimensions of visual hierarchical stimuli by humans (Homo sapiens), chimpanzees (Pan troglodytes), and baboon (Papio papio). In T. Matsuzawa (Ed.), Primate origins of human cognition and behavior (pp. 87-103). Tokyo: Springer-Verlag.

Herrnstein, R. J. (1984). Objects, categories, and discriminative stimuli. In H. L. Roitblat, T. G. Bever, \& H. S. Terrace (Eds.), Animal cognition (pp. 233-261). Hillsdale, NJ: Erlbaum.

Herrnstein, R. J. (1985). Riddles of natural categorization. In L. Weiskrantz (Ed.), Animal intelligence (Vol. 7, pp. 129-144). Oxford: Clarendon Press.
HerRnstein, R. J., \& Loveland, D. H. (1964). Complex visual concept in the pigeon. Science, 146, 549-551.

Herrnstein, R. J., Loveland, D. H., \& Cable, C. (1976). Natural concepts in pigeons. Journal of Experimental Psychology: Animal Behavior Processes, 2, 285-311.

Hollard, V. D., \& Delius, J. D. (1982). Rotational invariance in visual pattern recognition by pigeons and humans. Science, 218, 804-806.

Huber, L. (1994). Amelioration of laboratory conditions for pigeons (Columba livia). Animal Welfare, 3, 321-324.

Huber, L., \& Aust, U. (2001). The relevance of evolution, species comparison, color, and categorization for the object identity problem. Cahier de Psychologie Cognitive/Current Psychology of Cognition, 20, 221-229.

Huber, L., Aust, U., Michelbach, G., Ölzant, S., \& Nowotny, R. (1999). Limits of symmetry conceptualization in pigeons. Quarterly Journal of Experimental Psychology, 52B, 351-379.

JACOBS, G. H. (1993). The distribution and nature of color vision among the mammals. Biological Review, 68, 413-471.

JiTsUMORI, M. (1991). Do monkeys see meaningful objects in slide pictures? In A. Ehara, T. Kimura, \& International Primatological Society (Eds.), Primatologytoday (pp. 293-296). Amsterdam: Elsevier.

KirKPATricK, K. (2001). Object recognition. In R. G. Cook (Ed.), Avian visual cognition [on-line]. Retrieved from www.pigeon.psy.tufts.edu/ avc/kirkpatrick/.

Kirkpatrick-Steger, K., Wasserman, E. A., \& Biederman, I. (1996). Effects of spatial rearrangement of object components on picture recognition in pigeons. Journal of the Experimental Analysis of Behavior, 65, 465-475.

Kirkpatrick-Steger, K., Wasserman, E. A., \& Biederman, I. (1998). Effects of geon deletion, scrambling, and movement on picture recognition in pigeons. Journal of Experimental Psychology: Animal Behavior Processes, 24, 34-46.

Lea, S. E. G., \& DitTrich, W. (2000). What do birds see in moving video images? In J. Fagot (Ed.), Picture perception in animals (pp. 143180). Hove, U.K.: Psychology Press.

Loidolt, M., Aust, U., Meran, I., \& Huber, L. (2003). Pigeons use item-specific and category-specific information in the identification and categorization of human face stimuli. Manuscript submitted for publication.

Miller, R. J. (1973). Cross-cultural research in the perception of pictorial materials. Psychological Bulletin, 80, 135-150.

Mollon, J. D. (1989). "Tho' she kneel'd in that place where they grew ..." Journal of Experimental Biology, 146, 21-38.

NAVON, D. (1977). Forest before trees: The precedence of global features in visual perception. Cognitive Psychology, 9, 353-383.

NAvON, D. (1981). The forest revisited: More on global precedence. Psychological Research, 43, 1-32.

Navon, D. (1983). How many trees does it take to make a forest? Perception, 12, 239-254.

RILEY, D. A. (1984). Do pigeons decompose stimulus compounds? In H. L. Roitblat, T. G. Bever, \& H. S. Terrace (Eds.), Animal cognition (pp. 333-350). Hillsdale, NJ: Erlbaum.

Ryan, C. M. E., \& LEA, S. E. G. (1994). Images of conspecifics as categories to be discriminated by pigeons and chickens: Slides, video tapes, stuffed birds and live birds. Behavioural Processes, 33, 155-175.

SteEle, K. M. (1990). Configural processes in pigeon perception. In M. L. Commons, R. J. Herrnstein, S. M. Kosslyn, \& D. B. Mumford (Eds.), Quantitative analyses of behavior: Vol. 8. Behavioral approaches to pattern recognition and conceptformation (pp. 111-125). Hillsdale, NJ: Erlbaum.

Towe, A. L. (1954). A study of figural equivalence in the pigeon. Journal of Comparative Psychology, 47, 283-287.

Troje, N. F., Huber, L., Loidolt, M., Aust, U., \& Fieder, M. (1999). Categorical learning in pigeons: The role of texture and shape in complex static stimuli. Vision Research, 39, 353-366.

Van Hamme, L. J., Wasserman, E. A., \& Biederman, I. (1992). Discrimination of contour-deleted images by pigeons. Journal of Experimental Psychology: Animal Behavior Processes, 18, 387-399.

Vaughan, W. J., \& Greene, S. L. (1984). Pigeon visual memory capacity. Journal of Experimental Psychology: Animal Behavior Processes, 10, 256-271. 
Wasserman, E. A., Gagliardi, J. L., Cook, B. R., KirkpatrickSteger, K., Ast ley, S. L., \& Biederman, I. (1996). The pigeon's recognition of drawings of depth-rotated stimuli. Journal of Experimental Psychology: Animal Behavior Processes, 22, 205-221.

Wasserman, E. A., Kirkpatrick-Steger, K., Van Hamme, L. J., \& Biederman, I. (1993). Pigeons are sensitive to the spatial organization of complex visual stimuli. Psychological Science, 4, 336-341.

WATANABE, S. (1993). Object-picture equivalence in the pigeon: An analysis with natural concept and pseudo concept discrimination. Behavioural Processes, 30, 225-232.

Watanabe, S. (2000). How do pigeons see pictures? Recognition of the real world from its 2-D representation. In J. Fagot (Ed.), Picture perception in animals (pp. 71-90). Hove, U.K.: Psychology Press.

WATANABE, S. (2001). Discrimination of cartoons and photographs in pigeons: Effects of scrambling of elements. Behavioural Processes, $\mathbf{5 3}, 3-9$.

Watanabe, S., \& ITo, Y. (1991). Discrimination of individuals in pigeons. Bird Behavior, 9, 20-29.

Winner, H., \& EtTlinger, E. (1978). Do chimpanzees recognize photographs as representations of objects? Neuropsychology, 17, 413-420.

Wurm, L. H., Legge, G. E., Isenberg, L. M., \& LuebKer, A. (1993). Color improves object recognition in normal and low vision. Journal of Experimental Psychology: Human Perception \& Performance, 19, 899-911.

(Manuscript received September 6, 2002; revision accepted for publication February 12, 2003.) 